\title{
Angewandte
}

Supporting Information

for

Angew. Chem. Int. Ed. Z52389

(c) Wiley-VCH 2003

69451 Weinheim, Germany 


\section{Molecular Motion of Endohedral fullerenes in Single-Walled Carbon Nanotubes}

Andrei N. Khlobystov, Kyriakos Porfyrakis, Mito Kanai, David A. Britz, Arzhang Ardavan, Hisanori Shinohara, John Dennis and G. Andrew D. Briggs

\section{Production and purification of $i \mathrm{CeC}_{82}$}

Ce-containing endohedral fullerenes were produced by the so-called reversed arc technique developed by the Nagoya group as described previously. ${ }^{1}$ A Ce-doped graphite rod (anode) was kept a few mm apart from a graphite block (cathode) in a vacuum chamber. The rod and the block were connected to an external power supply and high current was passed through them $(300-500 \mathrm{~A})$. The vaporisation took place in He atmosphere $(50-100$ Torr $)$. The resulting soot contained $10-20 \%$ fullerenes.

The fullerenes were soxhlet-extracted from the soot for ca. 4 hours in o-xylene. A twostage high performance liquid chromatography (HPLC) method was employed to isolate individual fullerene isomers. In the first stage, the o-xylene solution was passed through a Cosmosil 5PBB column $(20 \times 250 \mathrm{~mm}$, Nacalai Tesque) with o-xylene eluent (flow rate: 11 $\mathrm{ml} / \mathrm{min}$ ). Figure 1 shows the HPLC chromatogram of Ce-containing endohedral fullerenes.

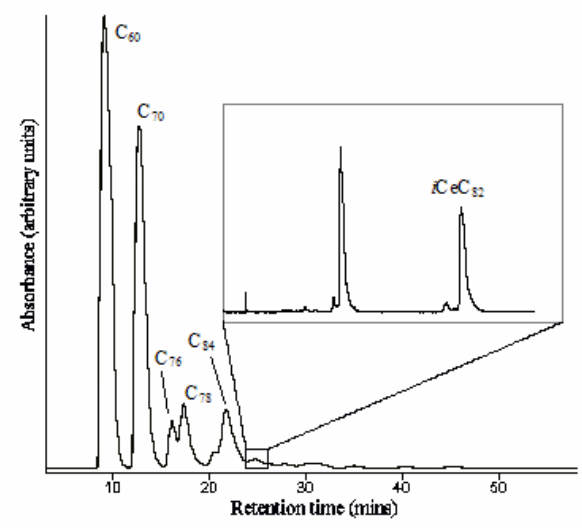

Figure 1. A HPLC chromatogram of Ce-containing endohedral fullerenes. The main diagram shows the first stage separation and, the inset shows the second (recycling) stage of $\mathrm{Ce} @ \mathrm{C}_{82} \cdot \mathrm{Ce}_{2} @ \mathrm{C}_{80}$ was isolated by using the same technique (not shown).

Mass spectrometry has shown that the peak between 24 and 28 min contains mainly $\mathrm{Ce} @ \mathrm{C}_{82}$ and $\mathrm{Ce}_{2} @ \mathrm{C}_{80}$.

A recycling HPLC technique was employed to isolate $\mathrm{Ce} @ \mathrm{C}_{82}$ and $\mathrm{Ce}_{2} @ \mathrm{C}_{80}$ from other empty fullerene species. The $24-28$ min fraction in the first stage was passed through a Cosmosil 5PYE column $(20 \times 250 \mathrm{~mm}$, Nacalai Tesque) with toluene eluent (flow rate: 18 $\mathrm{ml} / \mathrm{min}$ ) in recycling mode. After a few cycles, $\mathrm{Ce} @ \mathrm{C}_{82}$ was completely isolated, as shown in figure 1 (inset). Mass spectrometry has confirmed the purity of the material (figure 2). With the same method $\mathrm{Ce}_{2} @ \mathrm{C}_{80}$ was also purified. 


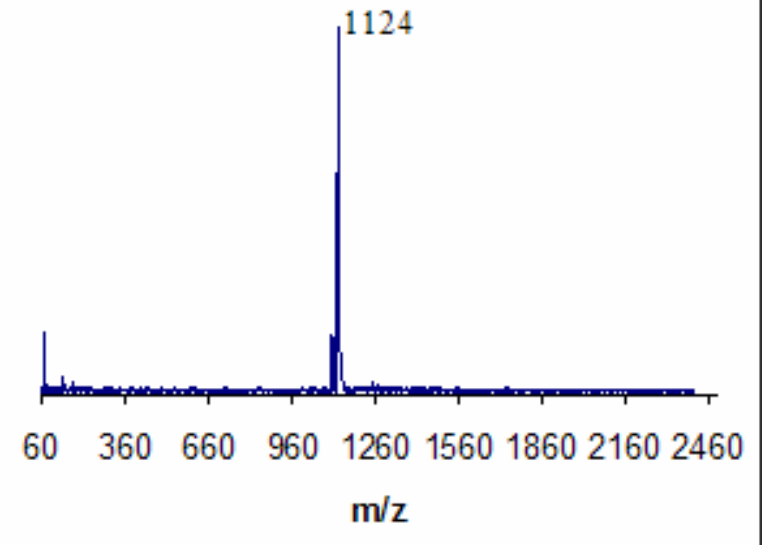

Figure 2. MALDI mass spectrum of the isolated Ce $@ \mathrm{C}_{82}$.

\section{Raman Spectra of SWNTs.}

Arc-purchased SWNTs (Aldrich) were used for the Raman experiments. Raman spectroscopy, conventionally employed for measurements of nanotube diameters, ${ }^{2}$ demonstrates the broad diameter distribution of the raw nanotubes (Figure 3a). The diameters have been calculated from the position of the radial breathing vibration mode (RBM) of SWNT according to the $\mathrm{d}_{\mathrm{NT}}=248 / \omega_{\mathrm{RBM}} \cdot{ }^{2}$ We have analysed the RBM profile of nanotubes in a broad range of excitation wavelengths. At all wavelengths we observed only two RBM peaks, although relative peak intensities change due to different bandgaps. This indicates that there are only two major diameters present in the sample after purification. the SWNT diameter distribution was reduced to two major diameters $15.1 \AA$ and $13.9 \AA^{3}$ (Figure $3 b$ ).

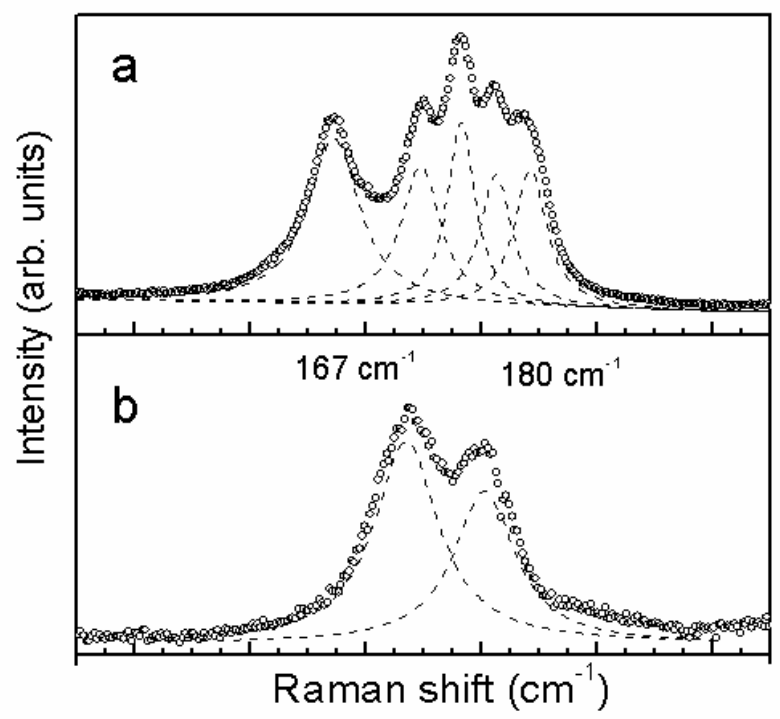

Figure 3. Raman spectra of (a) raw SWNTs and (b) purified SWNTs (excitation $\lambda=512 \mathrm{~nm}$ ). ${ }^{3}$

\section{X-Ray Energy Dispersive Spectroscopy}

The measurement of energy dispersive x-ray (EDX) spectra was performed using an electron microscope in a small probe mode with the electron probe size $\sim 3 \mathrm{~nm} \times 3 \mathrm{~nm}$. The spectra were recorded for isolated SWNTs bundles containing Ce@ $\mathrm{C}_{82}$ and confirmed the presence of 
Ce atoms inside the SWNTs. A typical EDX spectrum is shown in Figure 4. The large peaks of $\mathrm{Cu}$ and $\mathrm{Fe}$ are due to the specimen holder. Only the bottom part of the C-peak is shown (1$2 \mathrm{keV})$.

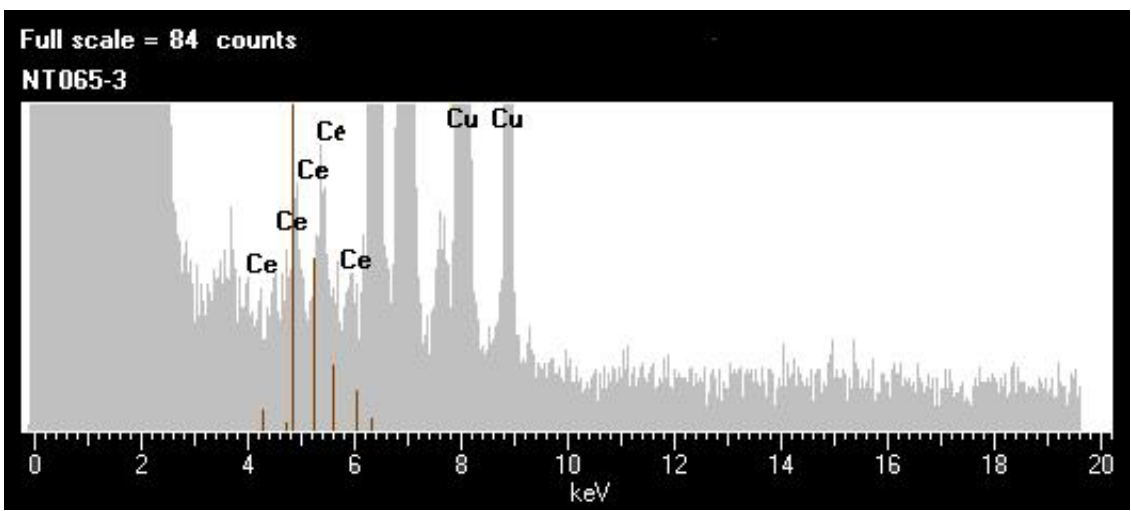

Figure 4. An EDX spectrum of $\left(\mathrm{Ce} @ \mathrm{C}_{82}\right) @ \mathrm{SWNT}$.

\section{Intermolecular interactions in $\left(\mathrm{Ce} @ \mathrm{C}_{82}\right) @$ SWNT systems}

Owing to the asymmetric charge distribution in the Ce@ $\mathrm{C}_{82}$, the interaction between the molecules depends strongly on the orientation of the fullerene cages (Figure 5). The electrostatic forces are likely to dominate the intermolecular interactions because of the high dipole moment.

a)

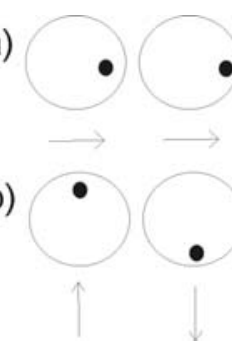

c)

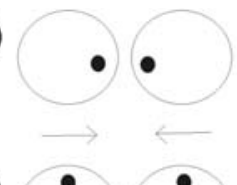

d)

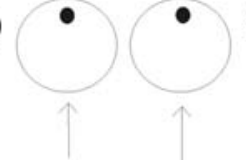

e)

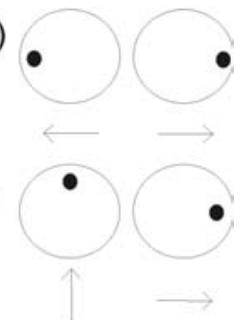

Figure 5. Schematic representation of orientations for pairs of $\mathrm{Ce} @ \mathrm{C}_{82}$ molecules. Directions of dipole moments are indicated by arrows assuming that $\mathrm{Ce}$-atoms in fullerene cages are located within the cross-section plane.

The position of the $\mathrm{Ce}^{3+}$ ion indicates the positive end of the molecular dipole. By examining separations between positively and negatively charged parts of $\mathrm{Ce} @ \mathrm{C}_{82}$ molecules, it is possible to deduce whether the electrostatic interaction has attractive or repulsive character. For example, orientations (a) and (b) shown in Figure 5 correspond to attractive interactions, orientations (c)-(e) correspond to repulsive interactions and orientation (f) corresponds to no electrostatic interaction between two molecules.

HRTEM imaging of $\left(\mathrm{Ce} @ \mathrm{C}_{82}\right) @ S W N T s$ allows visualisation of the Ce-atom positions, which can be used to determine the projections of molecular dipole moments on the plane perpendicular to the electron beam. 


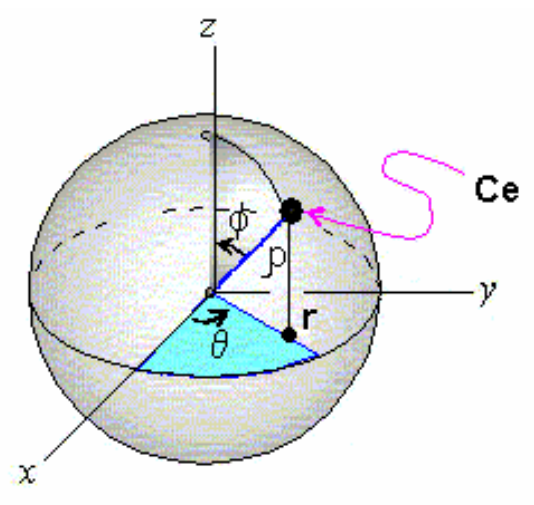

Figure 6. Spherical system of coordinates applied for $\mathrm{Ce} @ \mathrm{C}_{82}$.

The orientation of the Ce $@ \mathrm{C}_{82}$ molecule is most conveniently expressed in the spherical coordinates (Figure 6). In the absence of external electric fields, the $\mathrm{Ce}$ atom will be found with equal probability at any point of the surface of the sphere of constant diameter $\rho$. The plane of the HRTEM photograph coincides with the $x y$ plane where the projection of Ce-atom appears as a dark spot at a distance $\mathbf{r}$ from the centre of the fullerene cage. The direction of the dipole moment is defined by two angles $\theta$ and $\phi$. The angle $\theta$ can be measured directly from TEM images; the angle $\phi$ can be calculated as $\phi=\arcsin (r / \rho)$ so that angles $\phi$ and $180^{\circ}$ $\phi$ are indistinguishable. This precludes the direct determination of the dipole moment direction by HRTEM.

In order to reveal the mechanism of intermolecular interactions in nanotube Suenaga et al. have measured the distance between metal atoms of two neighbouring molecules (Figure 7a) as a single geometrical parameter describing dipolar interactions in $\left(\mathrm{La} @ \mathrm{C}_{82}\right) @$ SWNTs systems. ${ }^{4}$ An advantage for using $\mathrm{d}_{\mathrm{Ce}-\mathrm{Ce}}$ for statistical analysis is the fact that this parameter can be measured from HRTEM pictures. A similar approach used for $\left(\mathrm{Ce} @ \mathrm{C}_{82}\right) @$ SWNTs shows a distribution pattern similar to $i \mathrm{LaC}_{82} @ \mathrm{SWNT}$ with a maximum at $\mathrm{d}_{\mathrm{Ce}-\mathrm{Ce}}=13 \AA$ (Figure $7 b)^{4}$
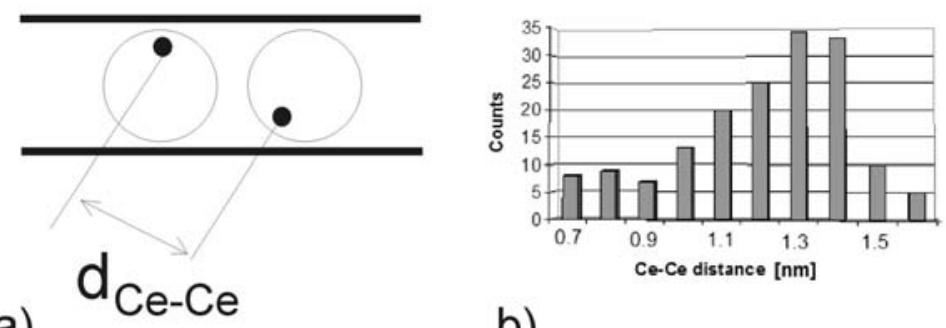

b)

Figure 7. (a) Schematic representation of two Ce@ $\mathrm{C}_{82}$ molecules, $\mathrm{d}_{\mathrm{Ce}-\mathrm{Ce}}$ is the distance between Ce-ions. (b) Distribution of Ce-Ce distances for pairs of neighbouring molecules in $\left(\mathrm{Ce} @ \mathrm{C}_{82}\right) @ \mathrm{SWNTs}$.

In reference 4, Suenaga et al. interpret a nonuniform distribution of the kind shown in Figure $7 \mathrm{~b}$ as evidence for electrostatic interactions controlling the fullerene orientations. However, we find that the probability distribution of $\mathrm{d}_{\mathrm{Ce}-\mathrm{Ce}}$ calculated for random orientations of $\mathrm{Ce} @ \mathrm{C}_{82}$ (Figure 8) has maximum in the same region as the experimental distribution (Figure $7 \mathrm{~b}$ ), indicating that this measurement is in fact insensitive to the intermolecular interactions. Spherical aberrations of the microscope and any deviation of the interfullerene separations from the van der Waals distance $(3.3 \AA)$ cause a greater uncertainty in $\mathrm{d}_{\mathrm{Ce}-\mathrm{Ce}}$ measurements than in angular parameter measurements (Figure 8). 
a)

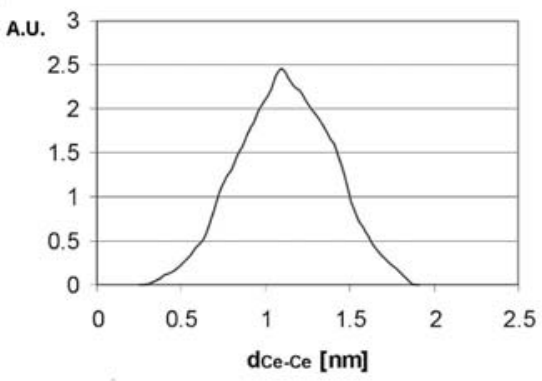

b)

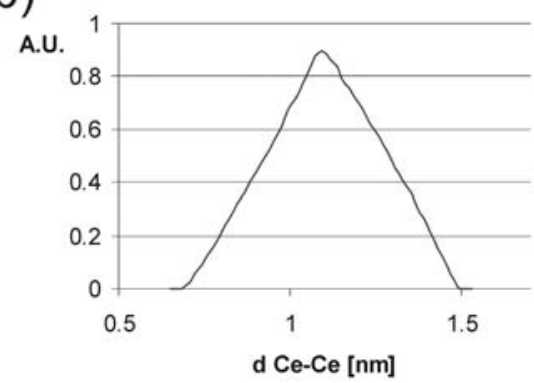

Figure 8. The probability distribution of $\mathrm{d}_{\mathrm{Ce}-\mathrm{Ce}}$ for an interfullerene spacing of $1.1 \mathrm{~nm}$; distance of Ce from centre of fullerene $0.4 \mathrm{~nm}$ (a) and $0.2 \mathrm{~nm}$ (b), in the absence of interfullerene interactions.

The fullerene orientation $\theta$ is another parameter that can be measured directly from HRTEM images. In the absence of interactions, the probability distribution function for angle $\theta$ is constant at any point (Figure 9c) owing to the spherical geometry of the system of coordinates (Figure 6). This leads to equal probability for any value of $\theta$ in isolated $\mathrm{Ce} @ \mathrm{C}_{82}$, and $\theta$ is thus a more appropriate parameter for statistical analysis than $\mathrm{d}_{\mathrm{Ce}-\mathrm{Ce}}$.
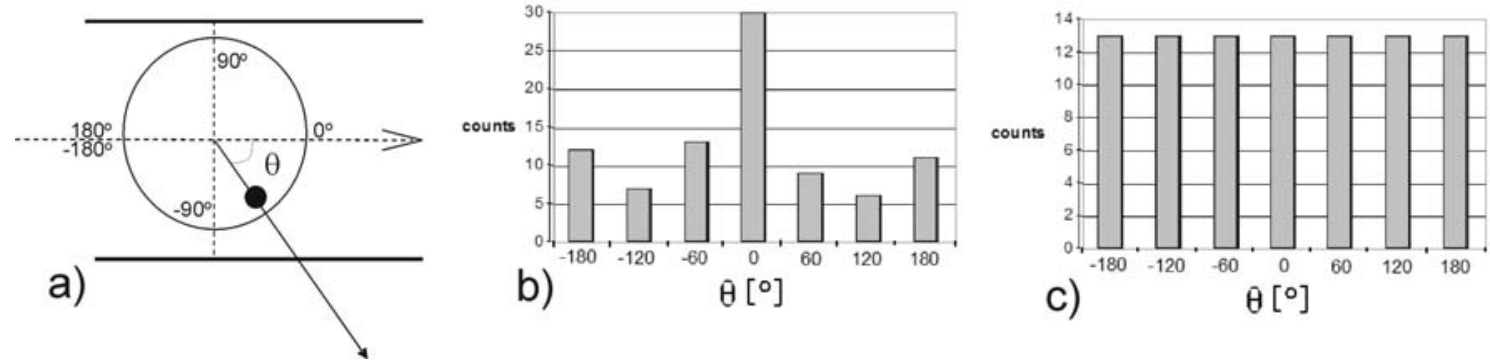

Figure 9. (a) Schematic representation of $M @ C_{n}$ inside a nanotube: the nanotube direction is shown by the dashed arrow, the dipole direction is shown by solid arrow, $\theta$ is the angle between these directions. (b) Experimental distribution of $\theta$ in $\left(\mathrm{Ce} @ \mathrm{C}_{82}\right) @$ SWNTs. (c) Theoretical distribution of $\theta$ in $\left(\mathrm{Ce} @ \mathrm{C}_{82}\right) @$ SWNTs for random orientations (no interactions).

We measured angles $\theta$ for $\mathrm{Ce} @ \mathrm{C}_{82}$ inside nanotubes with respect to the nanotube axis as shown in figure 9a. The observed distribution for $\theta$ (Figure 9b) indicates a preferential orientation for Ce@ $\mathrm{C}_{82}$ with the dipole moment nearly parallel to the nanotube axis (small angles $\theta$ ). Suenaga et al. ${ }^{4}$ have performed analogous statistical analysis for $\left(\mathrm{La} @ \mathrm{C}_{82}\right) @$ SWNTs systems showing a similar trend.

Comparison of angles $\theta_{1}$ and $\theta_{2}$ for pairs of neighbouring fullerenes shows a modest correlation between the orientations (Figure 10).

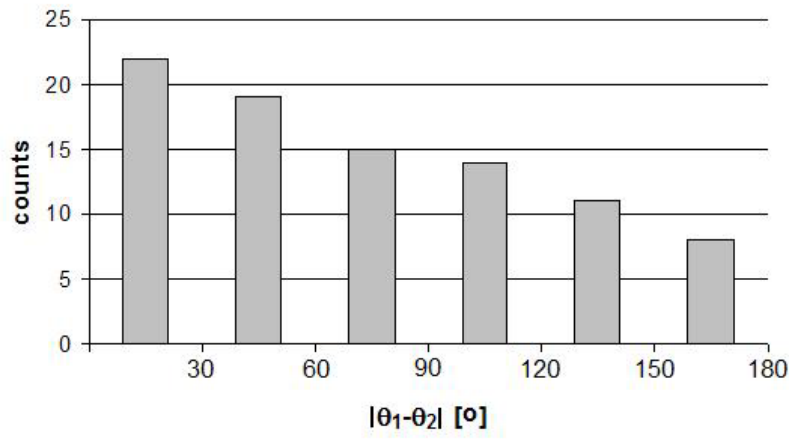

Figure 10. Experimental distribution of $\left|\theta_{1}-\theta_{2}\right|$ for pairs of neighbouring fullerenes in $\left(\mathrm{Ce} @ \mathrm{C}_{82}\right) @$ SWNTs.

The observation of a peak in the distribution of $\theta$ indicates that interactions of some kind (i.e. either between neighbouring fullerenes or between a particular fullerene and the tube) play a role in determining the fullerene orientation. The observation that $\left|\theta_{1}-\theta_{2}\right|$ is peaked at zero 
and is not bimodal (i.e. peaked at both $0^{\circ}$ and $180^{\circ}$ ) confirms that interfullerene interactions are important.

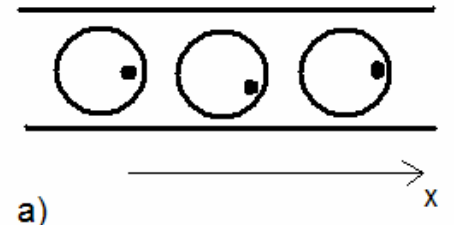

a)

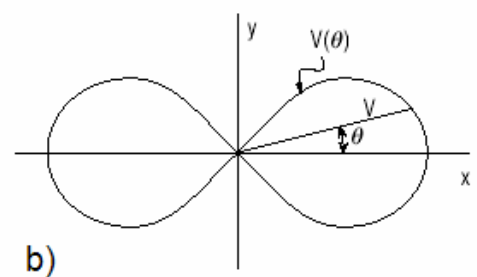

Figure 11. (a) Schematic representation of $M @ C_{n}$ molecules lined up in a nanotube and (b) angular dependence of the dipole potential for points separated by a constant distance from the centre of the dipole ( $\mathbf{r}$ is constant, $\theta$ is varying).

A candidate mechanism for interfullerene interactions is the electrostatic dipolar interaction. Each molecule in $\left(\mathrm{M} @ \mathrm{C}_{\mathrm{n}}\right) @$ SWNTs experiences the local electrostatic field determined by two nearest neighbours constrained by the nanotube to form a chain (Figure 11a). The electrostatic interaction between $\mathrm{Ce} @ \mathrm{C}_{82}$ molecules is strongest when the dipoles are lined up within the chain (Figure 11a). Therefore, the preferential molecular orientation observed for $\mathrm{Ce} @ \mathrm{C}_{82}$ can be explained by the presence of electrostatic interactions in $\left(\mathrm{M} @ \mathrm{C}_{\mathrm{n}}\right) @ \mathrm{SWNTs}$ systems. The identification of the exact mechanism of these interactions requires more precise experimental data and more advanced statistical analysis, which will be reported elsewhere.

\section{Maximum transferable energy from electron beam to fullerenes.}

Most of the incident electrons in TEM are transmitted without interaction with the thin specimen such as an isolated nanotube. The mean free path of the electrons is $\sim 150 \mathrm{~nm}$ for carbon materials [5]. Collisions of electrons with atoms can be elastic or inelastic. Elastically scattered electrons do not transfer energy to the atoms and these electrons are used for the image formation in TEM. However, inelastic processes are likely to excite phonons in the specimen which may have a significant effect on the molecular motion in nano-peapod systems. The magnitude of the phonon excitation induced by the electron beam can be conveniently expressed, for example, through the increase the specimen temperature during TEM imaging.

Inelastic processes of high energy involving interactions of the e-beam with core electrons or with atomic nuclei of the specimen are followed by the large losses of the transferred energy via emission of X-rays or Auger electrons, or via plasmon oscillations [ref. 5 , page 9]. As a result only small amount of the beam energy is actually transferred to atomic and molecular vibrations. The direct excitation of phonons by the e-beam has a very low probability and the transferable energy for this inelastic process is $\sim 0.1 \mathrm{eV}$ [ref. 6 , page 61 ]. The specimen heating due to the combined effect of all inelastic processes is difficult to measure experimentally. However the temperature increase can be theoretically estimated to be $\sim 10 \mathrm{~K}$ for semiconducting materials and even less for metallic [7].

Smith and Luzzi report on electron radiation effects in SWNTs [8], and rule out phonon excitation as a possible mechanism for the beam-damage in SWNTs. Their detailed study indicates that the ballistic knock-on collisions play by far more important role for carbon nanomaterials than other mechanisms. The transferable energy for knock-on collisions, strongly dependant on the accelerating voltage of the microscope, is significantly minimised at HT $100 \mathrm{keV}$ as compared to the standard imaging conditions, and no e-beam damage occurs at voltages below $86 \mathrm{keV}$. The transferable energy from the knock-on collisions also depends on the composition of the molecule and the geometry of the collision. This energy for $\mathrm{Ce} @ \mathrm{C}_{82}$ molecule can be estimated to be $0.21 \mathrm{eV}$ using the ballistic mechanism described in reference 8 . This energy is higher than energy transferred via other 
inelastic mechanisms and, therefore, it can be considered as a maximum of transferable energy from the e-beam to $\mathrm{Ce} @ \mathrm{C}_{82}$.

Electrical charging of specimens is a common problem for insulating materials (i.e. ceramics, oxides) when transmission electron microscopy is used for their characterisation. The electrons of e-beam can be locally trapped in the insulating specimens, which may cause serious difficulties for microscopic characterisation. However, carbon nanotubes are intrinsically superb heat and charge conductors, as demonstrated by numerous conductivity measurements on bulk as well as isolated nanotubes. Specifically, all SWNTs used for our experiments are either metallic or narrow-bandgap semiconductors as indicated by optical measurements (near IR-visible range). Moreover, several research groups have recently demonstrated that the presence of incar-fullerenes inside semiconducting nanotubes can make their band gap as narrow as $0.1 \mathrm{eV}$. During HRTEM imaging only a small area of a nanotube or a nanotube bundle is exposed to the e-beam. This area is in the region of $50 \times 50 \mathrm{~nm}^{2}$ in our microscope. Because the average nanotube length in this specimen is about 3-4 microns, the possible charge induced by the e-beam in a small part of the nanotube rapidly dissipates along the nanotube and will be absorbed by the copper grid used for mounting the sample inside the microscope. These facts allow concluding that the charging effect in carbon nanotubes is negligible.

\section{References:}

1. H. Shinohara, Rep. Prog. Phys., 2000, 63, 843.

2. S. Bandow et al., Phys. Chem. Lett., 2001, 347, 23.

3. A.N. Khlobystov, R. Scipioni, G.A.D. Briggs, A. Ardavan, S.G. Lyapin, D.A. Britz, D. Mnguyen, D. Pettifor, R.J. Nicholas, submitted.

4. K. Suenaga et al., Appl. Phys. A, 2003, 76, 445.

5. R. Brydson, Electron Energy Loss Spectroscopy, Microscopy Handbook v. 48

6. (BIOS, Scientific Publishers Ltd, 2001). D.B. Williams, C.B. Carter, Transmission Electron Microscopy: A Textbook for Materials Science (Plenum, New York, 1996).

7. L.W. Hobbs in Introduction to Analytical Electron Microscopy (eds. J.J. Hren, J.I. Goldstein, D.C. Joy, Plenum Press, 1979).

8. B.W. Smith, D.E. Luzzi, J. Appl. Phys., 2001, 90, 3509. 